\title{
EFFECT OF GAMMA-IRRADIATION ON BIODEGRADABLE MICROSPHERES LOADED WITH RASAGILINE MESYLATE
}

\author{
MARCOS FERNÁNDEZ ${ }^{1,2^{*}}$, EMILIA BARCIA AND SOFÍA NEGRO ${ }^{1}$ \\ ${ }^{1}$ Department of Pharmaceutics, Faculty of Pharmacy, Complutense University of Madrid, Madrid 28040, Spain \\ ${ }^{2}$ Department of Pharmacy, Faculty of Pharmacy, University of Concepción, Concepción 4070386, Chile
}

\begin{abstract}
In the present study, the influence of gamma-irradiation was evaluated on the physicochemical characteristics and in vitro release of rasagiline mesylate (RM), a selective MAO-B inhibitor used in Parkinson's disease, from poly(D,L-lactide-co-glycolide) (PLGA) microspheres. Microspheres were prepared using PLGA 50:50 by the solvent evaporation technique (O/W emulsion). Microspheres were sterilized by gamma-irradiation and their influence was assessed by scanning electron microscopy (SEM), laser light diffraction, differential scanning calorimetry (DSC), X-ray diffraction (XRD), gel permeation chromatography (GPC), encapsulation efficiency (EE) and in vitro drug release. Gamma-irradiation of RM-loaded microspheres did not affect EE, DSC and XRD patterns. After gamma-irradiation, changes on the surface were observed by SEM, but no significant difference in mean particle size was observed. GPC measurements showed a decrease in molecular weight of the polymer after five days of in vitro release. The similarity factor value between irradiated and non-irradiates microspheres was $<50$, indicating the non-similarity of the release profiles. The sterilization technique had an effect on the integrity of polymeric system, significantly affecting in vitro release of RM from PLGA microspheres. Therefore, from our results we conclude that gamma-irradiation is not a suitable sterilization procedure for this formulation.
\end{abstract}

KEYWORDS: Gamma-irradiation; Microspheres; PLGA; Rasagiline mesylate; Parkinson's disease.

\section{INTRODUCTION}

Parkinson's disease (PD) is a progresive neurodegenerative movement disorder that affects one every hundred persons above 60 years ${ }^{1}$. There is no cure for $\mathrm{PD}$, however therapies can improve the quality of life for many years ${ }^{2}$.

Rasagiline is a monoamine oxidase inhibitor (IMAO) with selectivity and specificity for MAO type B (MAO-B), which provides symptomatic relief as monotherapy and as adjunctive therapy ${ }^{3}$. Rasagiline exhibits a potential disease-modifying effect for the $1 \mathrm{mg}$ dose $\mathrm{e}^{4}$. Rasagiline is rapidly absorbed by the gastrointestinal tract $\left(\mathrm{T}_{\max } 30 \mathrm{~min}\right)$. However, the oral bioavailability is only $36 \%$, with a very short elimination half-life $(0.6-2 \mathrm{~h})^{5}$. These biopharmaceutic and pharmacokinetics characteristics and the fact that rasagiline is used in a chronic treatment, make rasagiline a suitable candidate for the development of a controlled release system (microspheres). Microspheres elaborated with biodegradable and biocompatible polymers, such as poly-d,l-lactic-co-glycolic acid (PLGA) ${ }^{6,7}$, can achieve prolonged pharmacologic effects whereas lowering undesirable side-effects, also have the advantage of disappearing from the body once they have exerted their therapeutic effect ${ }^{8}$.

PLGA-microspheres intended for parenteral administration have to meet the pharmacopoeial requirements of sterility. For terminal sterilization of PLGA formulations, $\gamma$-irradiation is considered the method of choice ${ }^{9}$, and it has been successfully employed for sterilization of biodegradable PLGAmicrospheres intended for parenteral use ${ }^{10-14}$. Nevertheless, radiosterilization have some drawbacks. Fragmentations of covalent bonds and production of free radicals could be induced by the energy transfer occurring in the process thereby resulting in damage of the irradiated material. For this reason, the use of $\gamma$-irradiation as a sterilization method for medical products requires accurate analyses, to verify that the active molecule has not been modified and no toxic products have been produced.

Gamma-sterilization has been extensively used on biodegradable polymers and it has been indicated that after exposure to $\gamma$-irradiation polyesters undergo chain scission and crosslinking ${ }^{15}$. The ionizing radiation on biodegradable polyesters reduces the molecular weight in a dose-dependent manner, mainly by the formation of reactive radicals that accelerates polymer degradation rate ${ }^{16}$.

In the present study, the influence of $\gamma$-irradiation was evaluated on the physicochemical characteristic and in vitro release of RM from PLGA microspheres.

\section{EXPERIMENTAL}

\section{CHEMICALS}

Rasagiline mesylate (RM) was obtained from Hangzhou Onion Chemical Co Limited (China). Resomer ${ }^{\circledR} 502$ was obtained from Boehringer Ingelheim
Chemicals Division (Germany). Polyvinyl alcohol (PVA) Mw 72000 Da was purchased from Merck (Germany). Water was purified by Milli-Q filtration system (Millipore, USA) and it was used in the preparation of buffers and solutions. All reagents and solvents used in the experiments were of analytical nature and purchased from Panreac (Barcelona, Spain).

\section{Preparation of RM-loaded PLGA microspheres}

Microspheres were prepared by an oil-in-water $(\mathrm{O} / \mathrm{W})$ emulsion solvent evaporation method, according to a previously reported technique ${ }^{17}$. The amounts of RM and PLGA 502 used for the preparation of microspheres were $40 \mathrm{mg}$ and $400 \mathrm{mg}$, respectively. Briefly, the organic phase was prepared by dissolving PLGA in $1 \mathrm{ml}$ of $\mathrm{CH}_{2} \mathrm{Cl}_{2}$ and then dispersing $40 \mathrm{mg}$ of $\mathrm{RM}$ in the PLGA solution. The aqueous phase consisted of $5 \mathrm{ml}$ PVA ( $1 \% \mathrm{w} / \mathrm{v}, \mathrm{pH} 10)$. Both phases were emulsified with a polytron ${ }^{\circledR}$ system (RECO Kinematika GmbH PT 3000, Germany) for $60 \mathrm{~s}$ at $2500 \mathrm{rpm}$. The O/W emulsion was poured into a $100 \mathrm{ml}$ of PVA solution $(0.1 \% \mathrm{w} / \mathrm{v} \mathrm{pH} 10)$ with $5 \% \mathrm{w} / \mathrm{v} \mathrm{NaCl}$ and continuously stirred for $3 \mathrm{~h}$ at room temperature with a magnetic stirred to allow evaporation of the organic solvent. Then, the microspheres were vacuum-filtered through $5-\mu \mathrm{m}$ membrane filters, washed three times with deionised water and freeze-dried (Flexy-Dry ${ }^{\mathrm{TM}}$, FTS Systems, US) for at least $12 \mathrm{~h}$. The lyophilized microspheres were kept in a desiccator until use. Blank PLGA microspheres were also prepared.

\section{Sterilization by $\gamma$-irradiation}

RM-loaded PLGA microspheres (ten different batches) and blank PLGA microspheres were placed in 1-ml glass vials. They were labelled and packed surrounded with dry ice into a polyurethane container as described above $\mathrm{e}^{10}$, assuring low temperatures $\left(<5^{\circ} \mathrm{C}\right)$ during the irradiation process to prevent a possible acceleration of the hydrolytic degradation of PLGA. The samples were irradiated using ${ }^{60} \mathrm{Co}$ as the radiation source in the g- irradiation unit at Aragogamma S.A. (Barcelona, Spain). In accordance with the USP recommendations, a dose of $25 \mathrm{kGy}$ was used and, thereafter all formulations were characterized.

\section{Characterization of the RM-loaded PLGA microspheres}

Determination of RM-loading efficiency

RM was extracted from PLGA microspheres and then quantified by a RPHPLC method, previously developed and validated by the authors ${ }^{18}$.

Morphological characterization and size distribution

Microspheres morphology was examined by scanning electron microscopy (SEM, Jeol, JSM-6400, Japan). The samples were dried and gold sputtercoated before observation by SEM at $20 \mathrm{kV}$.

Particle size and size distribution were measured by laser light diffraction 
analysis (Microtrac-S3500, Microtrac, USA). The lyophilized microspheres were suspended in Milli-Q water and sonicated for $30 \mathrm{~s}$ before each determination to prevent clumping. Results are described in terms of mean diameter as well as standard deviation. Monodispersity of the microspheres populations was described quantitatively using the particle size dispersal coefficient (Span) calculated according to Span $=\left(\mathrm{D}_{90}-\mathrm{D}_{10}\right) / \mathrm{D}_{50}$, where $\mathrm{D}_{\mathrm{n}}$ $(\mathrm{n}=10,50$, and 90$)$ denotes the particle diameter at $10 \%, 50 \%$ and $90 \%$ of the volume distribution. A large Span is indicative of a more heterogeneous size distribution. Microsphere populations can be considered as monodispersed for Span values lower than $0.4^{19}$. Samples were analyzed in triplicate. Blank PLGA microspheres were also analyzed.

\section{Differential scanning calorimetry (DSC)}

DSC analysis was performed with a Mettler 820 DSC analyzer (Mettler Toledo, Switzerland). Samples $(5-10 \mathrm{mg}$ ) were sealed into aluminium pans and heated in nitrogen atmosphere (flow rate $40 \mathrm{ml} \mathrm{min}^{-1}$ ). An empty aluminium pan was used as reference. Samples were scanned from $-10^{\circ}$ to $250^{\circ} \mathrm{C}$ at a heating rate of $10^{\circ} \mathrm{C} \mathrm{min}{ }^{-1}$. Under these conditions, DSC thermograms were undertaken for RM, PLGA, blank PLGA microspheres and RM-loaded PLGA microspheres.

\section{$X$-ray diffraction $(X R D)$}

XRD diffractograms were obtained by means of an automatic powder X-PERT MPD Philips diffractometer combined with a high temperature chamber (Anton Paar HTK 10) with a Pt heating filament, Ni-filtered $\mathrm{Cu}-\mathrm{Ko}$ radiation $\left(\lambda=1.54056 \AA\right.$ ), a $2 \theta$ interval configuration, angle range $5-50^{\circ}$, scan step size $0.04^{\circ}$ and time per step 1s. Under these conditions, samples of RM, PLGA, blank PLGA microspheres and RM-loaded PLGA microspheres were assayed.

\section{In vitro release studies}

In vitro release assays were performed by suspending microspheres (20 $\mathrm{mg}$ ) into $3 \mathrm{ml}$ of PBS (sink conditions) at $\mathrm{pH} 7.4$ in a water shaker bath (NE5, Clifton, UK) at $37^{\circ} \mathrm{C}$ under constant agitation (100 strokes per minute). At predetermined time intervals samples were centrifuged at $6000 \mathrm{~g}$ for $5 \mathrm{~min}$ and the supernatant was extracted, filtered through $0.45-\mu \mathrm{m}$ filters and replaced with the same volume of fresh medium. Quantification of RM was performed by the HPLC method indicated before ${ }^{18}$. In vitro release tests were performed in triplicate for each batch of microspheres (10 batches).

\section{Gel permeation chromatography (GPC)}

Microspheres were dissolved in tetrahydrofuran $(4 \mathrm{mg} / \mathrm{ml})$. After filtration (PTFE filter, pore size $0.22 \mathrm{~mm}$, Teknokroma, Spain), $20 \mathrm{ml}$ of the solution were injected in a PLgel MIXED-D column $(5 \mathrm{~mm}, 7.5 \times 300 \mathrm{~mm}$, Varian, UK). All measurements were performed at a flow rate of $1 \mathrm{ml} / \mathrm{min}$ at room temperature with a Waters 1525 binary HPLC pump. The refractive indexes were measured using a 2414 refractive index detector (Waters, MA, US). Molecular weights were calculated by the system calibration software using narrow polystyrene reference materials of known molecular weights: 43700 , 18600, 6520, 2950, 1100 and 381 Da (Waters Corporation, Polymer Standard Service $\mathrm{GmbH}$, Germany). The molecular weights are indicated as weightaverage molecular weight (Mw) and number-average molecular weight (Mn). Triplicate samples of irradiated and non-irradiated microspheres at preparation and after five days of the in vitro release assay were analysed.

\section{Data analysis}

Results are expressed as mean \pm standard deviation (SD). The statistical significance of the different parameters was tested by one-way analysis of variance (ANOVA).

The similarity factor $\left(f_{2}\right)$ was calculated to compare mean release profiles of RM from sterilized and non-sterilized microspheres according to the following equation ${ }^{20}$ :

$$
\mathrm{f}_{2}=50 \log \left\{\left(1+(1 / \mathrm{n}) \sum_{\mathrm{t}=1}^{\mathrm{n}}(\mathrm{NSt}-\mathrm{St})^{2}\right)^{-0.5} \times 100\right\}
$$

where:

$\mathrm{n}$ is the number of time points.

NSt is the percentage of RM released from non-sterilized microspheres.

$\mathrm{St}$ is the percentage of RM released from sterilized microspheres.

\section{RESULTS AND DISCUSSION}

PLGA-microspheres intended for parenteral administration have to meet the pharmacopoeial requirements of sterility. Sterilization of PLGA delivery systems by means of ethylene oxide has some drawbacks since these polymeric systems are highly sensitive to moisture and high temperatures and have toxicological problems due to chemical residues ${ }^{21}$. On the other hand, the chemical lability of the active constituents and polymeric matrix materials generally limits the strategies employed for obtaining and acceptable sterile product to either an aseptic process, or terminal sterilization by $\gamma$-irradiation of the final product ${ }^{22}$. However, $\gamma$-radiation as a form of electromagnetic radiation, characterized by high penetration at a very low dose rate, can modify the performance of the drug delivery system, prolonging the peroxidative radiolytic mechanism due to the exposure time ${ }^{23}$. For instance, degradation products of the polymers can modify the aqueous environmental conditions thereby resulting in alterations of the $\mathrm{pH}$ inside the dosage forms ${ }^{24}$. As PLGA degradation is catalyzed by protons, this micro $\mathrm{pH}$ decrease can lead to autocatalytic effects and, accelerate polymer degradation ${ }^{25}$. However, it is possible that this acidic microenvironment is not formed due to the diffusion of monomeric and/or oligomeric acids out of the system to the release medium and/or the incoming hydroxide ions from the release medium into the system, avoiding any autocatalytic effect ${ }^{6}, 26$. All these events take place as a function of the size and porosity of the microspheres. Moreover, degradation products of the polymers can crystallize within the dosage form thereby resulting in a modification of porosity with these degradation products subsequently dissolving and diffusing out of the microspheres. Gamma-irradiation of polyesters can result in chain scission and molecular weight reduction ${ }^{6,27,28}$

The influence of $\gamma$-irradiation on the characteristics of the formulation in our study was investigated using a variety of techniques including SEM, laser light diffraction, DSC, XRDP and GPC. RM-loading efficiency and in vitro release of RM from sterilized and non-sterilized PLGA microspheres were also determined.

Sterilization of RM loaded microspheres by $\gamma$-irradiation induced modifications of surface morphology, which were easily detected by SEM. As shown in Figure 1, the surface of non-irradiated microspheres (Figure 1.1.a) is very smooth in comparison to treated samples: microphotographs of $\gamma$-irradiated samples (Figure 1.1.b) show irregular surfaces with small pores. The presence of pores was also evident inside the polymeric matrix of $\gamma$-irradiated microspheres (Figure 1.1.c) thereby indicating that the drug inside the microsphere can escape more easily when in contact with an aqueous medium.

This increase in surface roughness and porosity caused no modification in particle size distribution of irradiated microspheres. Particle size distribution of $\gamma$-irradiated microspheres (mean particle size $105.3 \pm 54 \mu \mathrm{m}$ ) practically overlapped that of non-irradiated particles (Figure 1.2.a and 1.2.b). Moreover, the sterilization procedure induced a slight aggregation of the microspheres, which correlates with the Span value obtained (1.83). Gamma-irradiation did not statistically affect $(p<0.05)$ the EE of RM into PLGA microspheres $(51.3 \pm 2.6 \%)$
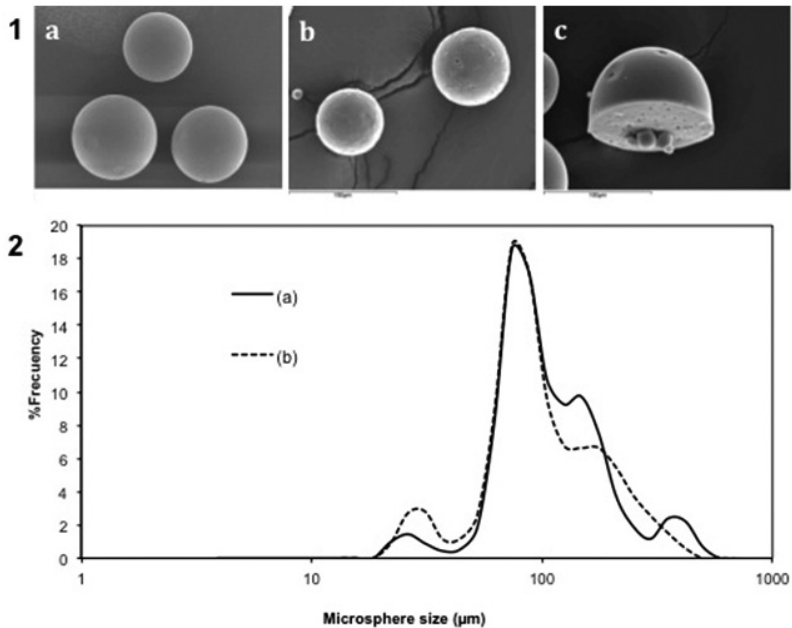

Figure 1. 1.1: SEM microphotographs of RM-loaded PLGA microspheres (non-irradiated) (a), RM-loaded PLGA microspheres ( $\gamma$-irradiated) (b) and transversal cross-section of RM-loaded PLGA microspheres ( $\gamma$-irradiated) (c) (x500). 1.2: Size distribution of RM-loaded microspheres (non-irradiated) (a), RM-loaded microspheres ( $\gamma$-irradiated) (b). RM: rasagiline mesylate. 
After XRD analysis the strongest maxima of intensity of non-irradiated RM were obtained at $10.42^{\circ}, 20.86^{\circ}, 25.18^{\circ}$ and $27.94^{\circ}$ being characteristic of its crystalline state (Figure 2a). These maxima were maintained after sterilization (Figure 2b). XRD of blank PLGA microspheres before and after sterilization were both similar showing the amorphous sate of PLGA (Figure $2 \mathrm{c}, 2 \mathrm{~d}$ and $2 \mathrm{e}$ ). In the patterns corresponding to RM-loaded PLGA microspheres before and after $\gamma$-irradiation (Figure $2 \mathrm{f}$ and $6 \mathrm{~g}$ ) the maxima of crystalline RM was not showed. It is reasonable to conclude that the solvent evaporation technique employed in microsphere formation might have resulted in a loss of crystallinity of the drug inside the microspheres. This loss of crystallinity could be explained by the fact that part of the drug is in a crystalline phase within the polymer matrix. In such crystalline phase, the drug has to be solubilized in the medium thereby prolonging its release from the microspheres.

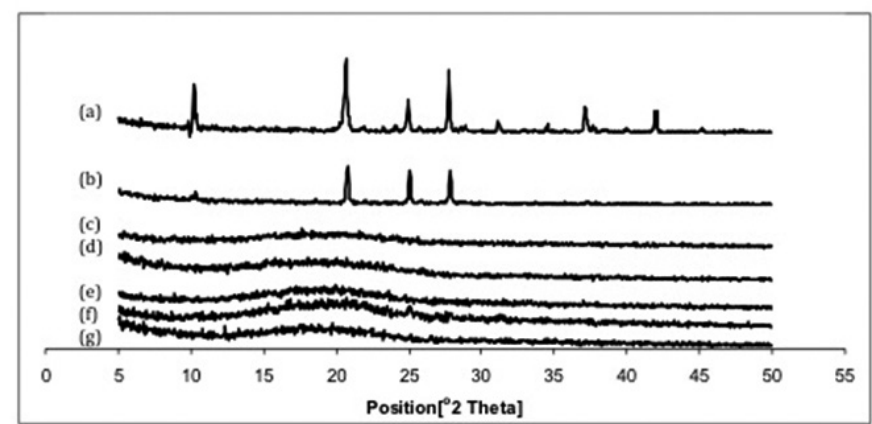

Figure 2: X-ray diffraction patterns of RM (a); $\gamma$-irradiated RM (b); PLGA (c); blank PLGA micospheres (d); $\gamma$-irradiated blank PLGA microspheres (e); RM-loaded PLGA microspheres (f); and $\gamma$-irradiated RM-loaded PLGA microspheres (g). RM: rasagiline mesylate.

In order to assess any possible interactions between RM and PLGA, DSC analysis was performed on RM, PLGA, blank microspheres and, RMloaded PLGA microspheres (Figure 3). DSC thermograms of RM before and after sterilization showed melting endothermic peaks at practically the same temperature $\left(217.4^{\circ} \mathrm{C}\right.$ and $214.3^{\circ} \mathrm{C}$, respectively). Thermograms of nonirradiated blank microspheres $\left(\mathrm{T}_{\mathrm{g}}=48.7^{\circ} \mathrm{C}\right)$ and PLGA $\left(\mathrm{T}_{\mathrm{o}}=51.1^{\circ} \mathrm{C}\right)$ also showed similar glass transition temperatures. Gamma-irradiation decreased the $\mathrm{T}_{g}$ values of both blank microspheres $\left(43.3^{\circ} \mathrm{C}\right)$ and RM-loaded PLGA microspheres $\left(41.2^{\circ}\right)$. These decreases could be explained by changes in the copolymer as a consequence of radiolytic events such as chain scissions ${ }^{29}$. DSC thermograms of both non-sterilized and sterilized RM-loaded PLGA microspheres showed melting endotherms at $211.2^{\circ} \mathrm{C}$, values slightly lower than those obtained for RM, probably due to an ionic interaction between amino groups of RM and the terminal carboxylic anions of the polymer ${ }^{30}$.

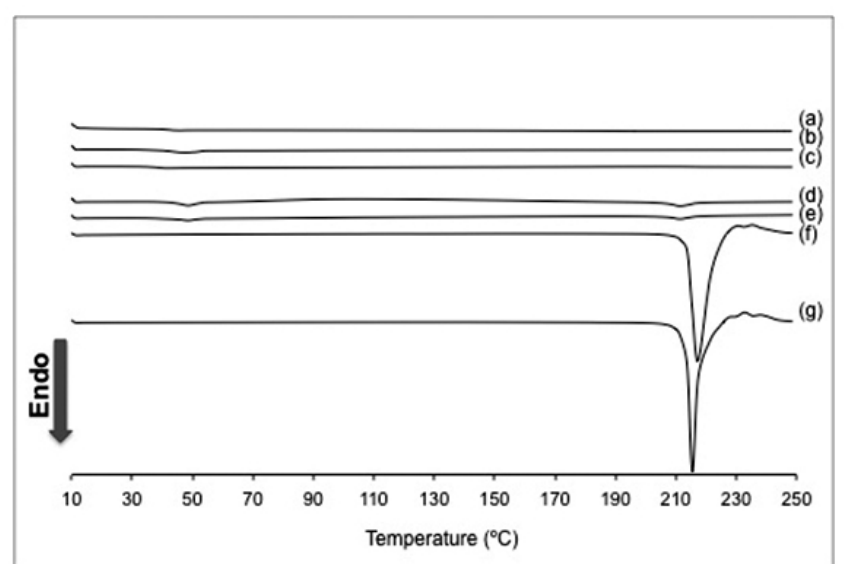

Figure 3: DSC thermograms of PLGA (a); blank PLGA micospheres (b); $\gamma$-irradiated blank PLGA microspheres (c); RM-loaded PLGA microspheres (d) ; $\gamma$-irradiated RM-loaded PLGA microspheres (e); RM (f); and $\gamma$-irradiated $\mathrm{RM}(\mathrm{g})$. RM: rasagiline mesylate.

GPC analysis (Table 1) was performed on RM-loaded PLGA microspheres before and after $\gamma$-irradiation being the analyses performed at the beginning and after five days of in vitro release. Weight-average molecular weight (Mw) and number-average molecular weight $(\mathrm{Mn})$ were determined. Gamma-irradiation of RM-loaded PLGA microspheres did not significantly affect both Mw and $\mathrm{Mn}$ at time zero of the release tests. However, after five days of in vitro release it was found that both $\mathrm{Mw}$ and $\mathrm{Mn}$ decreased when exposing RM-loaded PLGA microspheres to $\gamma$-irradiation. It can be observed that Mn decreased faster than Mw. Vollant et al. ${ }^{16}$ investigated the effects of increasing doses of $\gamma$-irradiation on captopril-loaded PLGA microspheres. The irradiation dose affected the in vitro release of captopril. This behaviour suggests that once in an aqueous medium the content of monomers and oligomers significantly increased in irradiated polymers and that $\mathrm{Mn}$ was more sensitive than Mw towards $\gamma$-irradiation. They hypothesized a cleavage mechanism which primary affected the terminal groups of polymer chains, hence the denomination of "unzipping mechanism", causing a faster decay of the Mn compared to Mw.

Figure 4 also shows the mean in vitro release profiles of RM from nonirradiated and irradiated PLGA microspheres. As it can be seen, initial burst release $(1 \mathrm{~h})$ is not influenced by the sterilization technique used; however, from this time point release of RM from sterilized microspheres was significantly increased. For instance, after 7 days $79 \%$ and $55 \%$ of RM was released from irradiated and non-irradiated microspheres respectively, thereby indicating that the sterilization technique significantly modified the release characteristics of RM from the microspheres. After two weeks of in vitro release approximately $90 \%$ of RM was released from both, non-irradiated and irradiated PLGA microspheres. Calculation of the similarity factor $\left(\mathrm{f}_{2}\right)$ between both release profiles gave a value lower than 50 (37.47), thereby indicating that the release profiles cannot be considered similar. Other authors have also shown that $\gamma$-irradiation affected the drug release rate ${ }^{31}$. For instance PLGA decomposition and the decrease in the average molecular weight increased both the extent and the rate of bupivacaine released from PLGA microspheres ${ }^{32}$.

Some authors have indicated that if the drug is dispersed in a solid drug delivery system, as in our case, the radiolysis of certain components of the formulation may be affect the drug release from the system and therefore, its efficacy and safety ${ }^{22,33}$.

Experimental studies on drug release from microspheres have shown that the process can be significantly affected by $\gamma$-irradiation ${ }^{34,35}$, with other reports in which in vitro drug release from microparticulate systems is not affected by $\gamma$-irradiation ${ }^{6,36}$. In our case, it has been demonstrated that $\gamma$-irradiation of RMloaded PLGA microspheres significantly modifies the release characteristics of the drug therefore; this sterilization procedure could not by applied for the final sterilization of the formulation due to the detrimental effects on the polymer.

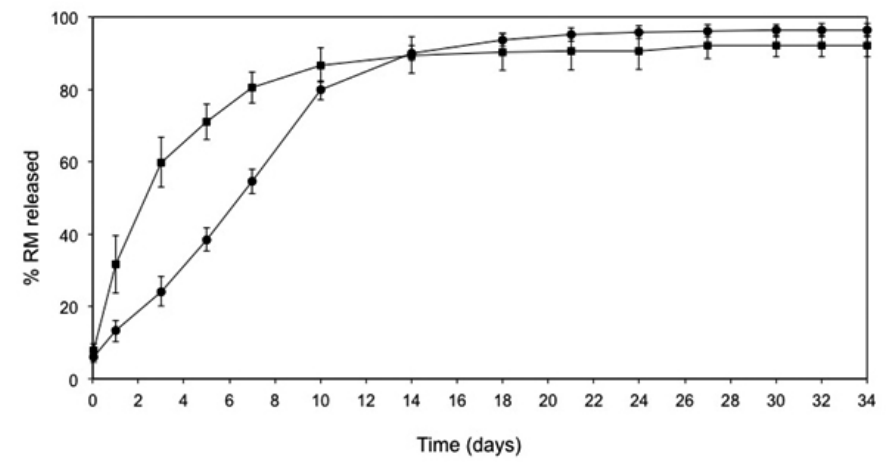

Figure 4: Mean release profiles of RM from non-irradiated $(\bullet)$ and $\gamma$-irradiated (घ) microspheres. RM: rasagiline mesylate.

\section{CONCLUSION}

The sterilization process by $\gamma$-irradiation at a dose of $25 \mathrm{kGy}$, affected no characteristics of loading efficiency, DSC and XRD patterns. However, the sterilization technique affected in vitro release of RM from PLGA microspheres. Therefore, from our results we conclude that $\gamma$-irradiation is not a suitable sterilization procedure for this formulation. The microspheres preparation will be make over aseptic processing. 
Table 1: GPC data of non-irradiated and irradiated RM-loaded PLGA microspheres (mean $\pm \mathrm{SD}, \mathrm{n}=3$ ) at time zero and after five days of in vitro release.

\begin{tabular}{|c|c|c|c|c|c|c|}
\hline \multirow{2}{*}{ Microspheres } & \multicolumn{2}{|c|}{ Mw } & \multicolumn{2}{c|}{ Mn } & \multicolumn{2}{c|}{ Mw/Mn } \\
\hline & Time zero & 5 days & Time zero & 5 days & Time zero & 5 days \\
\hline Non-irradiated & $17071.5 \pm 126.6$ & $16632.5 \pm 0.7$ & $12161.0 \pm 157.0$ & $12080.5 \pm 437.7$ & 1.40 & 1.38 \\
\hline$\gamma$-irradiated & $16536.5 \pm 116.7$ & $15088.0 \pm 33.9$ & $11999 \pm 5.7$ & $10217.0 \pm 234.8$ & 1.38 & 1.48 \\
\hline
\end{tabular}

\section{ACKNOWLEDGEMENTS}

The work was financially supported by a research project Fundación Mutua Madrileña (AP2696/2008) and the Complutense University UCMCAM research group 910939. Marcos Fernández thanks the support given by the Conicyt Program Fondecyt Iniciación N 11130387 and University of Concepción, Chile.

\section{REFERENCES}

1. D. Tarsy, JAMA 307, 2305 (2012).

2. B. Connolly, A. Lang, JAMA 311, 1670 (2013).

3. M. Youdim, A. Gross, J. Finberg, Br. J. Pharmacol. 132, 500 (2001).

4. C.W. Olanow, O. Rascol, R. Hauser, P.D. Feigin, J. Jankovic, A. Lang, W. Langston, E. Melamed, W. Poewe, F. Stocchi, E. Tolosa E., ADAGIO Study Investigators, N. Engl. J. Med. 361, 1268 (2009).

5. J. Chen, D. Swope, K. Dashtipour, Clin. Ther. 29, 1825, (2007).

6. J. Anderson, M. Shive, Adv. Drug Deliver. Rev. 28, 5 (1997).

7. J. Choi, K. Seo, J. Yoo, J. Pharm. Inv. 42, 155 (2012).

8. J.P. Benoit, N. Faisant, M.C. Venier-Julienne, P. Menei, J. Control. Release 65, 285 (2000).

9. European Guideline 3AQ4a. 1992. "The Use of Ionising Radiation in the Manufacture of Medicinal Products (6/1992), Official Publications of the Communities": áhttp://www.ema.europa.eu/docs/en_GB/document library/Scientific_guideline/2009/09/WC500002918.pdfñ̄, cited $2 \overline{0}$ August, 2014.

10. C. Martínez-Sancho, R. Herrero-Vanrell, S. Negro, J. Control. Release 99 , 41 (2004).

11. A. Hausberger, R. Kenley, P. DeLuca, Pharm. Res. 12, 851 (1995).

12. L. Montanari, M. Constantini, E. Signoretti, L. Valvo, M. Santucci, M. Bartolomei, P. Fattibene, S. Onori, A. Faucitano, B. Conti, I. Genta, J. Control. Release 56, 219 (1998).

13. B. Bittner, K.Mäder, C. Kroll, H. Borchert, T. Kissel, J. Control. Release 59, 23 (1999).

14. R. Dorati, C. Colonna, M. Serra, I. Genta, T. Modena, F. Pavanetto, P. Perugini, B. Conti, AAPS PharmSciTech. 9, 718 (2008).

15. M. Gupta, V. Deshmukh, Polymer 24, 827 (1983).

16. C. Volland, M. Wolff, T. Kissel, J. Control. Release 31, 293 (1994).

17. M. Fernández, S. Negro, K. Slowing, A. Fernández-Carballido, E. Barcia, Int. J. Pharm. 419, 271 (2011).

18. M. Fernández, E. Barcia, S. Negro, J. Pharm. Biomed. Anal. 49, 1185 (2009).

19. X. Xiao, L. Chu, W. Chen, J. Zhu, Polymer 46, 3199 (2005)

20. V. Shah, Y. Tsong, P. Sathe, L. Jen-Pei, Pharm. Res. 15, 889 (1998).

21. M. Claybourn, H. Gray, D. Murphy, I. Purnell, C. Rowlands, J. Control. Release 91, 431 (2003).

22. S. Caliş, S. Bozdag, H. Kaş, M. Tunçay, A. Hincal, Farmaco 57, 55 (2002).

23. L. Woo, C. Sandford, Radiat. Phys. Chem. 63, 845 (2002)

24. A. Shenderova, T. Burke, S. Schwendeman, Pharm. Res. 16, 241 (1999).

25. M. Dunne, I. Corrigan, Z. Ramtoola, Biomaterials 21, 1659 (2000).

26. P. Sansdrap, A. Moës, J. Control. Release 43, 47 (1997).

27. M. Sintzel, K. Schwach-Abdellaoui, K. Mäder, R. Stösser, J. Heller, C. Tabatabay, R. Gurny, Int. J. Pharm. 175, 165 (1998).

28. J. Nijsen, A. van Het Schip, M. van Steenbergen, S. Zielhuis, L. KroonBatenburg, M. van de Weert, P. van Rijk, W. Hennink, Biomaterials 23, 1831 (2002).

29. L. Montanari, F. Cilurzo, F. Valvo, A. Faucitano, A. Buttafava, A. Groppo, I. Genta, B. Conti, J. Control. Release 75, 317 (2001).

30. H. Okada, Adv. Drug Deliver. Rev. 28, 43 (1997).

31. C. Carrascosa, L. Espejo, S. Torrado, J. Torrado, J. Biomater. Appl. 18, 95 (2003).
32. L. Montarani, F. Cilurzo, F. Selmin, B. Conti, I. Genta, G. Poletti, F. Orsini, L. Valvo, J. Control. Release 90, 281 (2003).

33. S. Yoshioka, Y. Aso, S. Kojima, J. Control. Release 37, 263 (1995).

34. G. Spenlehauer, M. Vert, J.P. Benoit, F. Chabot, M. Veillard, J. Control. Release 7, 217 (1988).

35. J. Ruiz, J. Busnel, J.P. Benoit, Pharm. Res. 7, 928 (1990).

36. S. Yoshioka, Y. Aso, T. Otsuka, S. Kojima, Radiat. Phys. Chem. 46, 281 (1995). 